\title{
Signposts of Multiple Planets in Debris Disks
}

\author{
Kate Y. L. Su and G. H. Rieke \\ Steward Observatory, University of Arizona, \\ 933 N Cherry Avenue, AZ 85750, USA \\ email: ksu@as.arizona.edu, grieke@as.arizona.edu
}

\begin{abstract}
We review the nearby debris disk structures revealed by multi-wavelength images from Spitzer and Herschel, and complemented with detailed spectral energy distribution modeling. Similar to the definition of habitable zones around stars, debris disk structures should be identified and characterized in terms of dust temperatures rather than physical distances so that the heating power of different spectral type of stars is taken into account and common features in disks can be discussed and compared directly. Common features, such as warm $(\sim 150 \mathrm{~K})$ dust belts near the water-ice line and cold $(\sim 50 \mathrm{~K})$ Kuiper-belt analogs, give rise to our emerging understanding of the levels of order in debris disk structures and illuminate various processes about the formation and evolution of exoplanetary systems. In light of the disk structures in the debris disk twins (Vega and Fomalhaut), and the current limits on the masses of planetary objects, we suggest that the large gap between the warm and cold dust belts is the best signpost for multiple (low-mass) planets beyond the water-ice line.
\end{abstract}

Keywords. circumstellar matter - infrared: stars, planetary systems

\section{Five Zones of Debris Dust}

Great advances are being made in the observations of debris disks using all available platforms, revealing enduring patterns in the behavior of debris disk structure and its evolution that point toward general aspects of planetary system information and development that are not accessible by any other means. Although each individual resolved disk appears to be different from the others, combining all multi-wavelength observations from many different ground- and space-based facilities, debris disk structures can be broadly characterized by five different zones at roughly four different temperatures with thermal emission peaked at four different wavelengths (see Fig. 1). A majority of disks posses dense, cold $(\sim 50 \mathrm{~K})$, Kuiper-belt-analog dust that emits prominently in the far-infrared near $70 \mu \mathrm{m}$. Closer to the star, $\sim 20 \%$ of the debris systems have a warm $(\sim 150 \mathrm{~K})$, asteroid-like-analog dust near the water-ice line (Ballering et al. 2013). Even closer, dust in the terrestrial zone has a temperature of $\sim 300 \mathrm{~K}$ with emission peaked around $10 \mu \mathrm{m}$. Some debris systems even have $\sim 1500 \mathrm{~K}$, very hot excess emission that has been detected through ground-based near-infrared interferometric techniques. Finally, the last zone is a large halo, an extended structure, surrounding the aforementioned four zones, that only contains small grains. Not all debris disks have these five zones. Furthermore, except for the one at the water-ice line, the temperatures of the zones and hence their spectral energy distributions (SEDs) depend on the exact locations of the leftover planetesimal belts and the shepherding planets. Therefore, the variation in these zones around different stars is probably related to the various paths by which a system forms and evolves.

Since the majority of debris systems are not spatially resolved, it is not trivial to convert the observed properties like measured dust temperatures to physical parameters like the location of planetesimal belts. The dust temperatures can be well constrained from well-sampled (both Wien- and Rayleigh-Jeans sides of the emission) disk SEDs from mid- to far-infrared. With an additional assumption that grains in a debris disk are 


\section{Five Zones of Debris Dust}

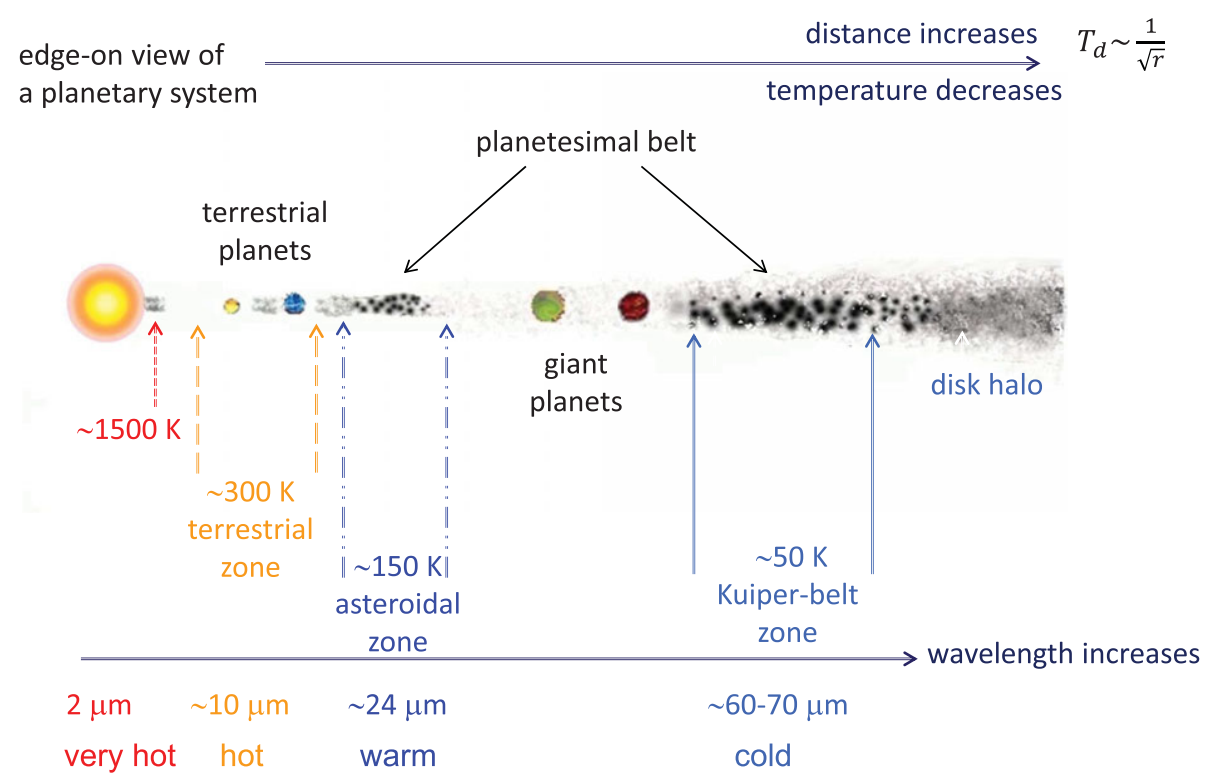

Figure 1. Illustration for the five zones of debris dust.

generated in collisional cascades that generally result in a steep size distribution (Gaspar et al. 2012), the observed (optically thin) disk emission is sensitive to the opacity of the disk, which is dominated by small grains. Ideally we can infer the location of the dust from the observed dust temperature and the average grain size in a disk and its optical properties. Using Spitzer IRS spectra and broad-band 70/100 $\mu \mathrm{m}$ photometry from Spitzer and Herschel, roughly a quarter of debris systems require two (warm and cold) temperatures to fit the disk emission. Although not all these two-temperature debris systems have two distinct dust belts, the ones that have resolved disk images at multiple wavelengths strongly suggest two separate planetesimal belts as we discuss below.

\section{Nearby Resolved Debris Disk Structures}

The HR 8799 System Among these two-temperature resolved disks, the most famous one is the HR 8799 (A5V) system, which has been under the spotlight since the discovery of the four massive planets by direct imaging (Marois et al. 2010). Besides the massive planets, the system also has a lot of dust generated from leftover planetesimals, predominantly at two characteristic dust temperatures: $\sim 150 \mathrm{~K}$ and $\sim 45 \mathrm{~K}$ ( $\mathrm{Su}$ et al. 2009). Figure 2 shows the disk images at 24 and $70 \mu \mathrm{m}$ along with its SED. At $24 \mu \mathrm{m}$, the emission is unresolved and dominated by the material closer to the star. At $70 \mu \mathrm{m}$, the disk is resolved as an elliptical ring with a position angle of $60^{\circ}$, indicating the system is slightly inclined by $10^{\circ}-25^{\circ}$ from face-on. From these images and dust temperatures measured from the SED, we can estimate the radial distances of these two belts. The four massive planets lie between the two dust belts as expected if planetary perturbations maintain their structures and create the dust free zone between them.

The Fomalhaut System Fomalhaut has a prominent cold $(\sim 50 \mathrm{~K})$ excess, dominated by dust generated in a narrow planetesimal belt at $\sim 140 \mathrm{AU}$, well resolved at multiple wavelengths (e.g. Kalas et al. 2005; Acke et al. 2012; Boley et al. 2012). The unresolved warm excess near the star was first discovered in the Spitzer $24 \mu \mathrm{m}$ image by 

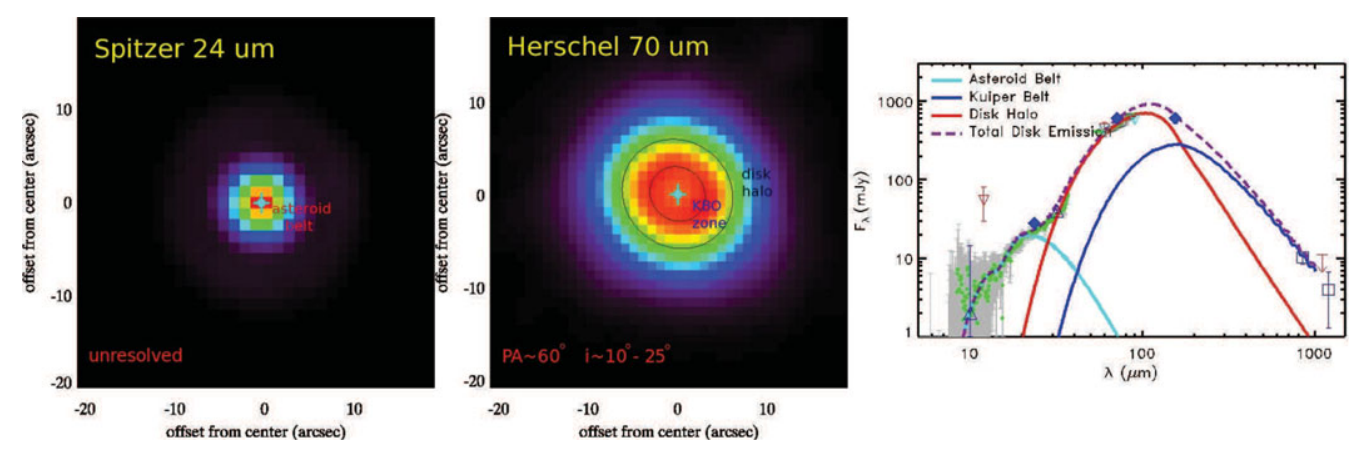

Figure 2. Multi-wavelength images of the HR 8799 system along with the disk SED.

Stapelfeldt et al. (2004), and later confirmed by Herschel at $70 \mu \mathrm{m}$ (Acke et al. 2012). Re-analysis of the Spitzer IRS spectrum centered at the star confirms that the unresolved excess emission arises from dust emission at a temperature of $\sim 170 \mathrm{~K}$, an asteroid-belt analog near the water-ice line ( $\mathrm{Su}$ et al. 2013). Using interferometic observations, the Fomalhaut system was found to possess a very hot K-band and hot N-band excesses at the levels of $0.88 \pm 0.12 \%$ and $0.35 \pm 0.10 \%$ above the photosphere (Absil et al. 2009; Mennesson et al. 2013). Recently, Lebreton et al. (2013) proposed these interferometric excesses are due to two populations of grains located at $\sim 0.1-0.3$ AU $(\sim 2000 \mathrm{~K})$ and $\sim 2$ AU $(\sim 400 \mathrm{~K})$. Although their model can explain the N-band null depths, the model SED significantly under-estimates the amount of the K-band excess (by 25\%), and over-estimates the excess in the IRS spectrum (i.e., $\gtrsim 3 \sigma$ for wavelengths of $15-30 \mu \mathrm{m}$ ).

The Vega System Vega and Fomalhaut are often referred to as debris disk twins since they are both A-type stars, located less than 8 pc away, half way through their main-sequence lifetime, and most importantly, they both have far-infrared excesses first discovered by IRAS 30 years ago, arising from dust particles in an enhanced Kuiper-belt analog. Su et al. (2013) present the Spitzer IRS spectrum taken at the star position and re-analysis of the Herschel $70 \mu \mathrm{m}$ image, and suggest the presence of an unresolved warm excess in the vicinity of the star with a dust temperature of $\sim 170 \mathrm{~K}$, roughly the temperature at which water ice sublimates. With other ancillary data, they confirm that Vega, just like Fomalhaut, also has an asteroid-belt analog.

Other Solar-like Systems $\epsilon$ Eri (K2V), like Fomalhaut and Vega, was found to possess a prominent far-infrared excess revealed by IRAS. Spitzer imaging and spectroscopic data combined with a detailed SED model further suggest a complex debris structure, with multiple zones in both warm $(\sim 150 \mathrm{~K})$ and cold $(\sim 50 \mathrm{~K})$ components (Backman et al. 2009). Using the same technique (SED and resolved cold component), the warm and cold configuration is also seen in the HD 107146 (Hughes et al. 2011) and HD 61005 (Hughes et al., this volume).

\section{Implications}

In our Solar System, the minor bodies that failed to form planets are arranged and sculpted by the planets over the course of $4.5 \mathrm{Gyr}$ of evolution. From knowledge of resonance structures observed in the Kuiper belt, we know that the outer giant planets have migrated in the past (e.g., Malhotra 1993) (not formed in-situ). The inner edge of the Kuiper belt's dusty disk is believed to be maintined by Neptune (Liou \& Zook 1999), whereas the outer edge of the Asteroid belt is maintained by the 2:1 orbital resonance with Jupiter (Kirkwood 1867; Dermott \& Murray 1983). Any debris system with similar structures is possibly signaling the presence of planets (e.g. the HR 8799 system). In 
the resolved systems discussed above, the orbital radius ratios $(\sim 10$, consistent with the estimated dust temperatures) between the inner warm and outer cold belts suggest a large gap in the minor-body population. This large, mostly dust-free zone may be maintained by one or multiple planet-mass objects through dynamical interaction, just like the HR 8799 system. For one single planet, the width of the chaotic zone depends on the location and eccentricity of the planet and its mass. Therefore, one can use the boundaries of the warm and cold dust belts to estimate the mass of the possible perturber.

We use the Vega system as an example where the warm belt is estimated at $\sim 11-14$ $\mathrm{AU}$ ( $\mathrm{Su}$ et al. 2013) and the cold belt is at $\sim 90-120 \mathrm{AU}$ ( $\mathrm{Su}$ et al. 2005, excluding the disk halo). For one single object on a circular orbit, the mass of the object has to be greater than $100 M_{J}$ (a brown dwarf), which should have been found in the intensive direct imaging searches (planets with masses $\gtrsim 3-4 M_{J}$ can be ruled out in the 20-70 AU range, Marois et al. 2006). For one single object on an eccentric orbit, the boundaries of the belts give an expected eccentricity of 0.8 (i.e., warm belt as the pericenter and cold belt as the apocenter). An object with such a high eccentricity inside the cold belt will have significant dynamical effects in the system, forcing the leftover planetesimals to be on highly eccentric orbits and creating an offset eccentric ring and/or resonance structures, which is not what is seen in the resolved disk images ( $\mathrm{Su}$ et al. 2013).

The giant planets are expected to form more efficiently outside the water-ice line, and these giant planets, once formed, likely experience inward or outward migration. The discovery of hot Jupiter systems in the early success of exoplanet searches is the best evidence for inward migration. Furthermore, if a giant planet did migrate inward, it is likely to destroy other bodies during the course of migration, consistent with the low incidence of warm excesses in such systems (Morales et al. 2012). Therefore, the existence of terrestrial planets and Asteroid belt in our Solar System is probably because it does not have a hot Jupiter. The same logic applies to these two-belt systems like Vega, Fomalhaut, $\epsilon$ Eri and HR 8799, having an asteroid belt might indicate a greater chance to harbor terrestrial planets compared to systems that do not have an asteroid belt.

\section{References}

Absil, O., Mennesson, B., Le Bouquin, J. -B., et al. 2009, ApJ, 704, 150

Acke, B., Min, M., Dominik, C., et al. 2012, A\&A, 540, 125

Backman, D., Marengo, M., Stapelfeldt, K. R., et al. 2009, ApJ, 690, 1522

Ballering, N. P., Rieke, G. H., Su, K. Y. L., \& Montiel, E. 2013, ApJ, 775, 55

Boley, A. C., Payne, M. J., Corder, S., et al. 2012, ApJL, 750, L21

Dermott, S. F. \& Murray, C. D. 1983, Nature, 301, 201

Gaspar, A., Psaltis, D., Rieke, G. H., \& Ozel, F. 2012, ApJ, 754, 74

Hughes, M., et al. 2011, ApJ, 740, 38

Kalas, P., Graham, J. R., \& Clampin, M. 2005, Nature, 435, 1067

Kirkwood, D. 1867, Meteoric Astronomy, co., 1867

Lebreton, J. et al. 2013, A\&A, 555, 146

Liou, J. C. \& Zook, H. A. 1999, AJ, 118, 580

Malhotra, R. 1993, Nature, 365, 819

Marois, C., Lafreniere, D. Doyon, R., et al. 2006, ApJ, 641, 556

Marois, C., Zuckeman, B., Konopacky, Q. M., et al. 2010, Nature, 468, 1080

Mennesson, B., Absil, O., Lebreton, J., et al. 2013, ApJ, 763, 119

Morales, F. Y., Padgett, D. L., et al. 2012, ApJ, 757, 7

$\mathrm{Su}, \mathrm{K}$. Y. L., et al. 2005, ApJ, 628, 487

$\mathrm{Su}, \mathrm{K}$. Y. L., Rieke, G. H., Stapelfeldt, K. R., et al. 2009, ApJ, 705, 314

$\mathrm{Su}, \mathrm{K}$. Y. L., Rieke, G. H., Malhotra, R., et al. 2013, ApJ, 763, 118 